\title{
Temporal characteristics of a comparator in adaptation to optical tilt ${ }^{1}$
}

\author{
SHELDON M. EBENHOLTZ ${ }^{2}$ AND GORDON M. REDDING \\ UNIVERSITY OF WISCONSIN, MADISON
}

Two groups of eight Ss each and one group of seven $S s$ were exposed to optical tilts $(T)$ of $50,40,30,20$, and 10 deg in succession. Exposure time at each tilt was 3, 15, and 27 min in Groups 1, 2, and 3, respectively. Trend analyses of the functions relating level of adaptation to $T$ showed significant quadratic components for Group 1, quadratic and linear components for Group 2, and only linear components for Group 3. These results were consistent with derivations from $a$ memory-comparator model of perceptual adaptation.

Several recent experiments (Ebenholtz, 1968, in press; Ebenholtz \& Mayer, 1968) have supported a theoretical model of the adaptation process based upon a comparator mechanism. In this model, the comparator is presumed to control changes in the level of adaptation (LA) by responding to the difference (D) between present and previous optical-tilt information (T). In the case of Ss exposed to several different degrees of prism tilt in succession, the output of the comparator, it may be assumed, reflects the entire set of differences that may be generated when comparing each of the preceding inputs with the present one. The further assumption that recent inputs have a greater effect upon the output of the comparator than do older ones, and that larger differences contribute more than do smaller ones, has led to a theoretical account of the relation between changes in LA and exposure time (Ebenholtz, 1969).

In principle, the assumption that differences based upon stored, i.e., previous, inputs contribute less and less to the LA the older they are, implies either some limit to the storage capacity or some type of process that interferes with the transfer of information of old inputs to the comparator. In the present experiment, the attempt was made to ascertain the amount of time over which stored inputs continue to contribute to the output of the comparator.

The present approach rests upon the finding that as $\mathbf{T}$ was decreased at 5 - $\mathrm{min}$ intervals in 8-deg steps from 40 to $8 \mathrm{deg}$ (Ebenholtz, 1969), LA first increased before decreasing. Thus, the function relating $\mathrm{LA}$ to exposure time, under conditions of stepwise reductions in tilt,
Table 1

The Set of Differences (D) Between Present and All Previous Inputs (T)

\begin{tabular}{lccccc}
\hline & \multicolumn{4}{c}{ Magnitude of $D($ Degrees) } \\
& $T=50$ & 40 & 30 & 20 & 10 \\
\hline$D_{1}$ & +50 & -10 & -10 & -10 & -10 \\
$D_{2}$ & & +40 & -20 & -20 & -20 \\
$D_{3}$ & & & +30 & -30 & -30 \\
$D_{4}$ & & & & +20 & -40 \\
$D_{5}$ & & & & & +10 \\
\hline
\end{tabular}

had a significant quadratic component. This outcome is deducible from the comparator model on the assumption that the preadaptation exposure period be considered equivalent to a $T$ of zero degrees and that differences from this value contribute, as do comparisons with other optical tilts, to the output of the comparator. The distribution of differences, with values of $T$ ranging from 50 to $10 \mathrm{deg}$, resulting from the comparison between ongoing, i.e., present, input and all preceding inputs is represented in Table 1. It may be noted that, since $\mathrm{T}$ decreases over time, all differences are negative with the exception of those produced by comparison with the 0 -deg input. The differences represented in columns beneath each ongoing prism tilt are ordered from most recent to oldest. Since the 0-deg input is always the oldest of the set, it may be expected to contribute positive differences but with a steadily diminishing effect on LA as the experiment progresses. Negative differences, however, increase in frequency and magnitude, and the net result to be expected is the nonmonotonic function relating LA to exposure time, as described above. The critical parameter in the present experiment was the amount of time between a given $T$ and the preceding $T$ values. The hypothesis tested was that, as this time interval was increased, comparisons with 0 deg would make a diminishing contribution to the output of the comparator. Manipulation of this variable, therefore, should produce a family of curves ranging from nonmonotonic, quadratic functions for small time intervals to monotonic, linear functions for large time intervals.

\section{METHOD}

Procedure

The Ss walked through an optically tilted hallway viewed with the right eye, the left eye being occluded. Tilt was produced by viewing through a set of right-angle prisms ${ }^{3}$ that could be rotated to any desired angle. Although the system produced a left-right reversal, adaptation to this transformation of the optic array was not measured. Prior to the start of the adaptation period and at five regular intervals thereafter, each $S$ was tested by setting a 12-in.-long, 1/4-in.-wide luminous line to the apparent vertical. Ss viewed the line with the previously exposed eye, without prisms, in a light-free room at a distance of $4 \mathrm{ft}$ and with head stabilized in a chin and forehead rest. At each test, $S$ made two judgments of the apparent vertical, with the starting position of the line placed altemately at $25 \mathrm{deg}$ clockwise (CW) and $25 \mathrm{deg}$ counterclockwise (CCW) of true vertical. The LA was defined in terms of the difference between the mean of the preadaptation settings and that obtained at any given test session.

\section{Design}

There were 23 paid volunteers, 8 each in two groups and 7 in the third. Alternate assignment of Ss into groups was not complete, and there was a total of $12 \mathrm{Ss}$ who could not complete the experiment because of nausea and dizziness that, in all likelihood, can be attributed to the right-left reversal of the prism system.

All groups were exposed to the identical set of prism tilts, starting at $50 \mathrm{deg}$ and decreasing to $10 \mathrm{deg}$ in $10-\mathrm{deg}$ steps. The groups differed in the amount of exposure time spent between shifts in $T$. Group 1 was exposed for $3 \mathrm{~min}$ at each value of $T$, whereas for Groups 2 and 3 , the interval between changes was 15 and $27 \mathrm{~min}$, respectively. Changes in $T$ were made at the end of each test session and without $S$ 's prior knowledge. In Groups 1 and 2, respectively, four Ss received CW rotations, the remaining Ss receiving $\mathrm{CCW}$ directions. In Group 3, four Ss were exposed to $\mathrm{CW}$ and three to $\mathrm{CCW}$ rotations.

\section{RESULTS}

In the analyses reported below, the data of Group 3 were considered to be based upon an $\mathbf{N}$ of 8 , and wherever appropriate, the mean of the distribution of seven $S$ s was treated as the score for the eighth $S$.

The three functions relating $L A$ to $T$ and indirectly to exposure time are represented 


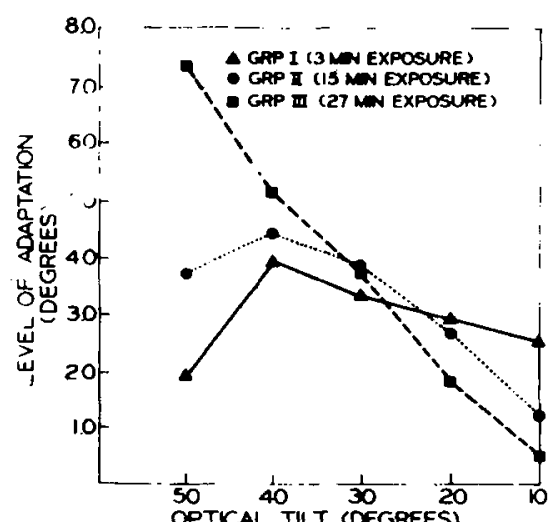

Fig. 1. Level of adaptation as a function of optical tilt, with exposure time as the parameter.

in Fig. 1. There were significant differences between groups trends $[F(8,84)=4.54$, $p<.01]$ attributable to differences among linear $[F(2,21)=6.26, p<.01]$ and quadratic components $[\mathrm{F}(2,21)=3.58$, $\mathrm{p}<.05]$, respectively. Individual trend analyses revealed, for Group 1, a significant quadratic component only $[F(1,7)=6.44$, $\mathrm{p}<.05]$. Thus, although the function, from $T=40$ to $T=10$, is apparently linear, the slope of this segment was not significantly different from zero. Group 2 yielded significant linear and quadratic components, $F(1,7)=36.82$ and 11.21 , respectively, with $p<.01$ in each case. Group 3 showed only a significant linear component $[F(1,7)=12.17, \mathrm{p}<.01]$.

\section{DISCUSSION}

As the exposure time at each value of $T$ was increased from 3 to 15 to $27 \mathrm{~min}$ for Groups 1, 2, and 3, respectively, the quadratic component of the function relating $L A$ to $T$ steadily diminished. When the shift in $T$ occurred every $27 \mathrm{~min}$, a linear function emerged. These results confurm the inferences drawn above from the comparator model and, therefore, indirectly support the basic assumptions of the model concerning the storage and comparison of information over time.

Since the function produced by Group 3 was linear, it can be concluded that stored inputs contribute little, if at all, to the output of the comparator, and hence to the LA, beyond about $27 \mathrm{~min}$, viz, the time interval between the 40-deg and the preexperimental 0 -deg tilt. This outcome is consonant with the assumption of a limited storage capacity such that traces of previous exposures are cleared from storage or forgotten. In the present context, this assumption requires the conclusion that information acquired in the "normal" or preadaptation state is lost within as short a time as $27 \mathrm{~min}$. This is, of course, counterintuitive and, in addition, actually is inconsistent with the finding that decay of adaptation occurs. That is, after reaching a given LA, subsequent measurements of adaptation with $S$ in a darkened room show a drop in LA in the direction of the preadaptation zero point (Ebenholtz, 1969). Since this change toward the preadaptation level occurs after time periods in excess of $27 \mathrm{~min}$, it cannot be concluded that the preadaptation direction has been lost within this interval. On the other hand, the fact of decay itself indicates that newly acquired directions are indeed lost over time. A possible resolution of this difficulty may be achieved by two assumptions. The first is that the rate of decay is related to the amount of exposure time during adaptation. One implication of this assumption is that even preadaptation states show decay, which may be indicated by an increase in variability of test judgments, but require longer decay intervals than those used previously. Magnitudes of the order of those used in sensory deprivation studies, i.e., 3 to 4 days, may be more appropriate to this point. The second assumption states that the longer the exposure time associated with more recent inputs, the more likely they are to interfere with the passage of information of old inputs from storage to the comparator. Thus, both decay and interference may contribute to the diminishing role of old inputs, relative to more recent ones, in determining the output of the comparator.

\section{REFERENCES}

EBENHOLTZ, S. M. Some evidence for a comparator in adaptation to optical tilt. Journal of Experimental Psychology, 1968, 77, 94-100.

EBENHOLTZ, S. M. Transfer and decay functions in adaptation to optical tilt. Journal of Experimental Psychology, 1969, 81, 170-173.

EBENHOLTZ, S. M., \& MAYER, D. Rate of adaptation under constant and varied optical tilt. Perceptual \& Motor Skills, 1968, 26, 507-509.

\section{NOTES}

1. This research was supported by Grant MH-13006-03 from the National Institu te of Mental Health, United States Public Health Service.

2. Address: Psychology Department, Psychology Building, Charter at Johnson Street, Madison, Wisconsin 53706.

3. The prisms were joined along the surface opposite the right angle with the line of sight, together with the axis of rotation, parillel to this surface.

(Accepted for publication July 16, 1969.) 